\title{
COMMUTATOR CALCULUS AND LINK INVARIANTS
}

\author{
K. T. CHEN
}

1. Introduction. Let $G$ be a finitely presented group such that the abelianized group $G /[G, G]$ has a basis of $n$ elements, which may possibly include elements of finite order. Let $G$ have a presentation of $n+k$ generators and $k+q$ relations. (Necessarily, $q \geqq 0$.) Then, for each integer $d>0$, a group \& presented by $n$ generators and $q$ relations may be constructed such that $\mathbb{S} / \mathcal{S}_{d}$ is isomorphic with $G / G_{d}$, where $\mathcal{S}_{d}$ and $G_{d}$ are the $d$ th lower central commutator subgroups of (5) and $G$ respectively. In the case that $G$ is the group of a link $L$ consisting of $n$ components, $B$ is a group presented by $n$ generators and $n$ relations. This is quite a helpful reduction in the number of generators and relations in the presentation of $G / G_{d}$, which determines the finitely generated abelian factor groups $G_{i} / G_{i+1}, i=1,2, \cdots, d$ -1 , and thus yields numerical invariants. This result is applied in $\$ 4$ to obtain a geometrical interpretation of the factor group $G / G_{3}$ of the group $G$ of a link $L ; G / G_{3}$ is completely determined by the number of components of $L$ and the linking numbers of the different pairs of components. In $\$ 5$ two examples are given. In one of them, it is shown that the torsion numbers of $G_{3} / G_{4}$ are sufficient to distinguish between a certain sequence of links, each of which has vanishing linking number for each pair of its three components. In the other example, it is shown that the torsion numbers of $G_{4} / G_{5}$ may be used to distinguish between another sequence of links of two components with vanishing linking number. However, for the group $G$ of a knot, the factor group $G / G_{d}$ is finite cyclic for every $d \geqq 2$. The author is obliged for the valuable suggestions of R. H. Fox and R. C. Lyndon.

2. Terminology and preliminary. For any group $G$, denote by $[a, b]$ the commutator $a b a^{-1} b^{-1}, a, b \in G$, and by $[A, B]$ the subgroup generated by all $[a, b], a \in A, b \in B$. Define inductively $\left[a_{1}\right]=a_{1}$, and for $d \geqq 1,\left[a_{1}, a_{2}, \cdots, a_{d+1}\right]=\left[\left[a_{1}, a_{2}, \cdots, a_{d}\right], a_{d+1}\right], a_{i} \in G$. Furthermore, set $G_{1}=G$ and, for $d \geqq 1, G_{d+1}=\left[G_{d}, G\right]$. The group $G_{d}$ is called the $d$ th lower central commutator subgroup of $G$ and is the normal subgroup generated by all $\left[a_{1}, a_{2}, \cdots, a_{d}\right], a_{i} \in G$.

Let $M$ be a normal subgroup of $G$. Then we write $a \equiv b \bmod M$ if and only if $a b^{-1} \in M$. Without ambiguity, we shall denote the cosets $a_{1} M, a_{2} M, \cdots$ by $a_{1}, a_{2}, \cdots \bmod M$. For any homomorphism

Received by the editors April 22, 1951. 
$\phi: G \rightarrow G^{\prime}, \phi\left(G_{d}\right) \subset G_{d}^{\prime}$, and, consequently, $a \equiv b \bmod G_{d}$ implies $\phi(a) \equiv \phi(b) \bmod G_{d}^{\prime}$.

It is known [5 $]^{1}$ that $u \in G_{s}, v \in G_{t}$ implies $[u, v] \in G_{s+t}$. By repeated use of this fact together with the identities

$$
\begin{aligned}
& {[a b, c]=a[b, c] a^{-1}[a, c],} \\
& {[c, a b]=[c, a] a[c, b] a^{-1},}
\end{aligned}
$$

we obtain the following lemma.

Lemma 1. If $a, a^{\prime} \in G_{s}, b, b^{\prime} \in G_{t}, a \equiv a^{\prime} \bmod G_{s+1}$, and $b \equiv b^{\prime} \bmod G_{t+1}$, then

$$
[a, b] \equiv\left[a^{\prime}, b^{\prime}\right] \bmod G_{8+t+1} .
$$

\section{The main theorem.}

MAIN Theorem. Let $G$ be a finitely presented group such that the abelianized group $G / G_{2}$ has a basis of $n$ elements. Suppose that $G$ is presented by $n+k$ generators and $k+q$ relations. (It is implied that $k \geqq 0$ and $q \geqq 0$.) Then, for each $d \geqq 0$, there is a group \&s presented by $n$ generators and $q$ relations such that

$$
G / G_{d} \cong G / G_{d}
$$

The case $q=0$ of this theorem has been proved by W. Magnus [6]. This section is devoted to a complete proof of this theorem, which will be restated later in a more constructive form (Theorem 1).

Let $G$ have a presentation

$$
G \cong\left\{\bar{a}_{i}, i=1,2, \cdots, n+k / \bar{r}_{i}, i=1,2, \cdots, k+q\right\},
$$

that is, $G$ is obtained from the free group $F$ generated by $\bar{a}_{i}, i=1,2, \cdots, n+k$, by introducing relations $\bar{r}_{i}\left(\bar{a}_{1}, \cdots, \bar{a}_{n+k}\right)$ $\equiv e, i=1,2, \cdots, k+q$. The elements $\tilde{a}_{i}$ also represent a set of generators of the abelianized group $G / G_{2}$, which has a basis of $n$ elements. We apply to the array $\left\{\bar{a}_{1}, \cdots, \bar{a}_{n+k}\right\}$ the following operation:

(A) $\bar{a}_{i}$ and $\bar{a}_{j}$ are interchanged,

(B) $\bar{a}_{i}$ is replaced by $\bar{a}_{i}^{t} \bar{a}_{j}^{m}, i \neq j, \epsilon= \pm 1$;

and to the array $\left\{\bar{r}_{1}, \cdots, \bar{r}_{k+q}\right\}$ the following operations:

(C) $\bar{r}_{i}$ and $\bar{r}_{j}$ are interchanged,

(D) $\bar{r}_{i}$ is replaced by $\bar{r}_{i}^{e} \bar{r}_{j}^{m}, i \neq j, \epsilon= \pm 1$.

Under these operations $\bar{a}_{1}, \cdots, \bar{a}_{n+k}$ and $\bar{r}_{1}, \cdots, \bar{r}_{k+q}$ will continue to be generators and relations for $G$ as well as for the abelian-

${ }^{1}$ Numbers in brackets refer to the bibliography at the end of the paper. 
ized group $G / G_{2}$. Let $r_{i} \equiv \prod_{j=1}^{n+k} a_{j}^{m_{i j}} \bmod F_{2}, i=1,2, \cdots, k+q$. To the operations (A), (B), (C), (D) there correspond elementary transformations on the matrix $\left\|m_{i j}\right\|$. From the classical theory of matrices, it follows that, after a finite number of operations (A), (B), (C), (D), the arrays $\left\{\bar{a}_{1}, \cdots, \bar{a}_{n+k}\right\}$ and $\left\{\bar{r}_{1}, \cdots, \bar{r}_{k+q}\right\}$ will become such that

(i) $\bar{a}_{1}, \cdots, \bar{a}_{n}$ form a basis of $G / G_{2}$,

(ii) $\bar{r}_{i} \equiv x_{i} \bar{a}_{n+i}^{-1} \bmod F_{2}, i=1,2, \cdots, k, x_{i}$ being a word in $\bar{a}_{1}, \cdots, \bar{a}_{n}$ alone,

(iii) $\overline{\boldsymbol{r}}_{k+i} \equiv e \bmod F_{2}, i=1,2, \cdots, q$.

Thus we are led to the following lemma.

Lemma 2. Let $G$ be defined as in the main theorem. Then $G$ has a presentation $G \cong\left\{a_{1}, \cdots, a_{n} ; b_{1}, \cdots, b_{k} / h_{1}, \cdots, h_{k} ; r_{1}, \cdots, r_{q}\right\}$ such that the relations $h_{i} \equiv e$ and $r_{i} \equiv e$ are in the following forms:

(i) $h_{i}=u_{i} x_{i} b_{i}^{-1}, u_{i} \in F_{2}, x_{i} \in \mathfrak{F}$,

(ii) $r_{i} \in F_{2}$,

where $F$ denotes the free group generated by $a_{i}, i=1,2, \cdots, n$, and $b_{i}, i=1,2, \cdots, k$, and $\mathfrak{F}$ denotes the free group generated by $a_{i}$, $i=1,2, \cdots, n$, alone.

We may assume hereafter in this section that $G$ has its presentation as given in this lemma. Observe that $a_{1}, \cdots, a_{n}$ form a basis in the abelianized group $G / G_{2}$. For simplicity, write the presentation of $G$ as $G \cong\{a, b / h, r\}$. Let $H$ be the normal subgroup generated by $h_{i}, i=1,2, \cdots, k$, and $R$ the normal subgroup generated by $r_{i}$, $i=1,2, \cdots, q$. Then $G \cong F / H \cdot R$.

Denote by $\psi(w)$ the word obtained from $w$ by replacing each $b_{i}$ by $u_{i} x_{i}$, and $\phi(w)$ the word obtained from $w$ by replacing each $b_{i}$ by $x_{i}, i=1,2, \cdots, k$. Then the substitution $\psi: F \rightarrow F$ is an endomorphism of $F$, and the substitution $\phi: F \rightarrow \mathfrak{F}$ is a homomorphism of $F$ onto $\mathfrak{F}$. Both $\psi$ and $\phi$ leave $\mathfrak{F}$ elementwise fixed, and $\phi^{2}=\psi \phi=\phi$. Moreover $h_{i}=\psi\left(b_{i}\right) b_{i}^{-1}$, and therefore $\psi(w) \equiv w \bmod H, w \in F$. We observe that, in the presentation $G \cong\{a, b / h, r\}$, to replace $r_{i}$ by $\psi\left(r_{i}\right)$ is a Tietze operation [8], and thus, by repeated use of $\psi$,

$$
G \cong\left\{a, b / h, \psi^{d-2}(r)\right\}, \quad d \geqq 2 .
$$

The notation $\psi^{d-2}(r)$ stands for the array $\psi^{d-2}\left(r_{1}\right), \cdots, \psi^{d-2}\left(r_{q}\right)$, and obvious notations of abbreviation similar to this will be often used. It will be an important technique in this paper to use the substitution $\psi$ as a Tietze operation on generators and relations of $G$.

Lemma 3. If $w_{t} \in F_{t}$, then 


$$
\psi^{d}\left(w_{t}\right) \equiv \phi \psi^{d-1}\left(w_{t}\right) \bmod F_{d+t},
$$

$d \geqq 1$.

Proof. For the generators $a_{i}$ and $b_{i}$ of $F$, we have $\psi\left(a_{i}\right)=a_{i}=\phi\left(a_{i}\right)$ and $\psi\left(b_{i}\right)=u_{i} x_{i} \equiv \phi\left(b_{i}\right) \bmod F_{2}$. It is thus true that, for $w \in F, \psi(w)$ $\equiv \phi(w) \bmod F_{2}$. We prove the lemma for $d=1$ by induction on $t$. Assuming that the lemma holds for $t-1, t \geqq 2$, it follows from Lemma 1 that $\psi\left(\left[w_{t-1}, w\right]\right)=\left[\psi\left(w_{t-1}\right), \psi(w)\right] \equiv\left[\phi\left(w_{t-1}\right), \phi(w)\right] \bmod F_{t+1}$ $\equiv \phi\left(\left[w_{t-1}, w\right]\right) \bmod F_{t+1}, w \in F, w_{t-1} \in F_{t-1}$. Since each element of $F_{t}$ is a product of commutators of the form $\left[w_{t-1}, w\right]$, we conclude that, for $w_{t} \in F_{t}, \psi\left(w_{t}\right) \equiv \phi\left(w_{t}\right) \bmod F_{t+1}$. The lemma now holds for $d=1$. Proceeding by induction on $d$, we assume that it holds for $d-1$ and any $t \geqq 1$. Then $\psi^{d-1}\left(w_{t}\right) \equiv \phi \psi^{d-2}\left(w_{t}\right)$ mod $F_{d+t-1}$, that is, $\psi^{d-1}\left(w_{t}\right)\left[\phi \psi^{d-2}\left(w_{t}\right)\right]^{-1} \in F_{d+t-1}$. By the validity of the lemma for $d=1$, $\psi\left(\psi^{d-1}\left(w_{t}\right)\left[\phi \psi^{d-2}\left(w_{t}\right)\right]^{-1}\right) \equiv \phi\left(\psi^{d-1}\left(w_{t}\right)\left[\phi \psi^{d-2}\left(w_{t}\right)\right]^{-1}\right) \bmod F_{d+t}$. Since $\psi \phi=\phi^{2}=\phi$, we have $\psi^{d}\left(w_{t}\right)\left[\phi \psi^{d-2}\left(w_{t}\right)\right]^{-1} \equiv \phi \psi^{d-1}\left(w_{t}\right)\left[\phi \psi^{d-2}\left(w_{t}\right)\right]^{-1}$ $\bmod F_{d+t}$. Hence

$$
\psi^{d}\left(w_{t}\right) \equiv \phi \psi^{d-1}\left(w_{t}\right) \bmod F_{d+t} .
$$

THEOREM 1. Let a group $G$ have a presentation as given in Lemma 2: $G \cong\{a, b / h, r\}$. Then the group $\mathbb{S} \cong\left\{a / \phi \psi^{d-3}(r)\right\}$ has the property

$$
\text { (\$) } / \mathcal{S}_{d} \cong G / G_{d}, \quad d \geqq 3 .
$$

REMARK. In the presentation of $\mathscr{S} \cong\left\{a / \phi \psi^{d-3}(r)\right\}$, each $r_{i}$ belongs to $F_{2}, i=1,2, \cdots, q$. If, for some $i, r_{i}$ belongs to $F_{t}, 2 \leqq t \leqq d-1$, then we may replace the corresponding $\phi \psi^{d-3}\left(r_{i}\right)$ by $\phi \psi^{d-1-t}\left(r_{i}\right)$ in the presentation of $\$ 5$.

Proof OF THE THEOREM. It follows from Lemma 3 that $\psi^{d-2}\left(r_{i}\right)$ $\equiv \phi \psi^{d-3}\left(r_{i}\right) \bmod F_{d}$, and from (2) that

$$
G / G_{d} \cong\left\{a, b / h, \psi^{d-2}(r), F_{d}\right\} ;
$$

consequently,

$$
G / G_{d} \cong\left\{a, b / h, \phi \psi^{d-3}(r), F_{d}\right\} .
$$

Notice that each $\phi \psi^{d-3}\left(r_{i}\right)$ belongs to $\mathfrak{F}$. Let $h_{i}^{\prime}=\phi \psi^{d-2}\left(b_{i}\right) b_{i}^{-1}$ and $h^{\prime}=\left\{h_{1}^{\prime}, h_{2}^{\prime}, \cdots, h_{\mathbf{k}}^{\prime}\right\}$. Since $\psi(w) \equiv w \bmod H, w \in F$, we have $\psi^{d-1}\left(b_{i}\right)$ $\equiv b_{i} \bmod H$ and, using Lemma $3, h_{i}^{\prime}=\phi \psi^{d-2}\left(b_{i}\right) b_{i}^{-1} \equiv \psi^{d-1}\left(b_{i}\right) b_{i}^{-1}$ $\bmod F_{d} \equiv e \bmod H \cdot F_{d}$. Therefore by Tietze operations we may introduce new relations $h_{i}^{\prime} \equiv e$ into the presentation of $G / G_{d}$ :

$$
G / G_{d} \cong\left\{a, b / h, h^{\prime}, \phi \psi^{d-3}(r), F_{d}\right\} .
$$

Now $\phi \psi^{d-2}$ is the substitution which replaces every $b_{i}$ in a word by $\phi \psi^{d-2}\left(b_{i}\right)$, and, due to the definition of $h^{\prime}$, we may replace $h$ by $\phi \psi^{d-2}(h)$ in this presentation of $G / G_{d}$ : 


$$
G / G_{d} \cong\left\{a, b / \phi \psi^{d-2}(h), h^{\prime}, \phi \psi^{d-3}(r), F_{d}\right\} .
$$

Observe that, due to Lemma $3, \psi^{d-1}\left(b_{i}\right) \equiv \phi \psi^{d-2}\left(b_{i}\right) \bmod F_{d}$, and $\phi \psi^{d-2}\left(h_{i}\right)=\phi \psi^{d-2}\left(\psi\left(b_{i}\right) b_{i}^{-1}\right)=\phi\left[\psi^{d-1}\left(b_{i}\right) \phi \psi^{d-2}\left(b_{i}\right)^{-1}\right] \equiv e \bmod F_{d}$. Again, by Tietze operations,

$$
G / G_{d} \cong\left\{a, b / h^{\prime}, \phi \psi^{d-3}(r), F_{d}\right\},
$$

and, using $h_{i}^{\prime} \equiv e$, that is, $b_{i} \equiv \phi \psi^{d-2}\left(b_{i}\right)$, as the defining relation of each $b_{i}$, we have

$$
G / G_{d} \cong\left\{a / \phi \psi^{d-3}(r), \mathfrak{F}_{d}\right\}
$$

Let $G \cong\left\{a / \phi \psi^{d-3}(r)\right\}$. Then

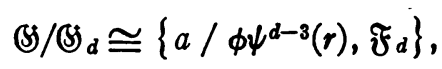

and hence the theorem is proved.

4. Application to link groups. A link is the union of $\boldsymbol{n}$ mutually disjoint, oriented, simple closed curves $L_{1}, \cdots, L_{n}$ in Euclidean 3 -space $E . L_{i}$ is called the $i$ th component of $L$. If each $L_{i}$ is a polygon, then $L$ is said to be polygonal. The fundamental group $G$ of the complement $E-L$ is called the group of the link $L$.

Through the well known Wirtinger method [7], we may read off a presentation of the group $G$ of a polygonal link $L$ through its regular projection. Let $G \cong\left\{a_{i j} / r_{i j}\right\}\left(i=1,2, \cdots, n ; j=1,2, \cdots, k_{i}\right)$ be such a presentation, where to each crossing point $Q_{i j}$ of the projection corresponds a relation $r_{i j} \equiv e, r_{i j}=b_{i j} a_{i j} b_{i j}^{-1} a_{i j+1}^{-1}=\left[b_{i j}, a_{i j}\right] a_{i j} a_{i j+1}^{-1}$ with $b_{i j}=a_{\alpha(i, j) \beta(i, j)}^{c_{i j}} .(\alpha(i, j)$ and $\beta(i, j)$ are given by the segment of $L$ which crosses over at $Q_{i j}$, and $\epsilon_{i j}= \pm 1$ is the signature of crossing.) $a_{i 1}, a_{i 2}, \cdots, a_{i k_{i}}$ are the Wirtinger generators corresponding to the segments (in their natural order) of the component $L_{i}$. The index $j$ on $a_{i j}, b_{i j}, \cdots$, and so on, is to be taken modulo $k_{i}$.

Define $a_{i}=a_{i 1}, v_{i j}=\left[b_{i j}, a_{i j}\right], r_{i}=v_{i k_{i}} v_{i k_{i}-1} \cdots v_{i 1}, u_{i 1}=e, i=1,2$, $\cdots, n, j=1,2, \cdots, k_{i}$. Let $u_{i j}=v_{i j-1} v_{i j-2} \cdots v_{i 1}, i=1,2, \cdots$, $n ; j=2,3, \cdots, k_{i}$. Define $h_{i j}=u_{i j} a_{i} a_{i j}^{-1}$. It may be straightforwardly verified that

$$
G \cong\left\{a_{i j} / h_{i j}, r_{i}\right\}, \quad i=1,2, \cdots, n ; j=1,2, \cdots, k_{i} .
$$

Each $r_{i}$ belongs to $F_{2}$. Define $\mathfrak{S} \cong\left\{a_{i} / \phi \psi^{d-3}\left(r_{i}\right)\right\}, i=1,2, \cdots, n$, where $d \geqq 3, \psi\left(a_{i j}\right)=u_{i j} a_{i}$, and $\phi\left(a_{i j}\right)=a_{i}$. Then, due to Theorem 1, we have $G / G_{d} \cong(\$) /\left(S_{d}\right.$.

In the case $d=3$, we have $\mathscr{S} \cong\left\{a_{i} / \phi\left(r_{i}\right)\right\}, i=1,2, \cdots, n$. Let $\mathfrak{F}$ be the free group generated by $a_{i}, i=1,2, \cdots, n$; then $\mathbb{G} / \mathbb{S}_{3}$

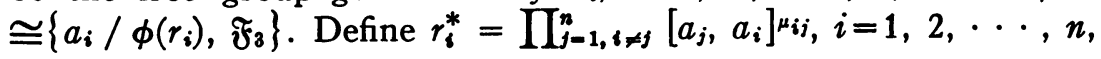


where $\mu_{i j}$ is the linking number of $L_{i}$ and $L_{j}$. Then $\phi\left(r_{i}\right)=\left[\phi\left(b_{i k_{i}}\right)\right.$, $\left.\phi\left(a_{i k_{i}}\right)\right] \cdots\left[\phi\left(b_{i 1}\right), \phi\left(a_{i 1}\right)\right]=\left[a_{\alpha\left(i_{i}, k_{i}\right)}^{e_{i k}}, a_{i}\right] \cdots\left[a_{\alpha(i, 1)}^{e_{i j}}, a_{i}\right] \equiv r_{i}^{*} \bmod \mathfrak{F}_{3}$ and $\mathbb{S} / \mathbb{S}_{3} \cong\left\{a_{i} / r_{1}^{*}, \mathfrak{F}_{3}\right\}$. Thus we have shown the following theorem.

Theorem 2. Let $L=L_{1} \cup \cdots \cup L_{n}$ be a polygonal link, and $G$ its group. Let $\mu_{i j}$ be the linking number of $L_{i}$ and $L_{j}, i \neq j$. Define $\mathbb{S}^{*}$ $=\left\{a_{i} / r_{i}^{*}\right\}$, where $i=1,2, \cdots, n$, and $r_{i}^{*}=\prod_{j=1, j \neq i}^{n}\left[a_{j}, a_{i}\right]^{\mu_{i j}}$. Then (S) $/ \mathcal{G S}_{3}^{*}$ is isomorphic with $G / G_{3}$.

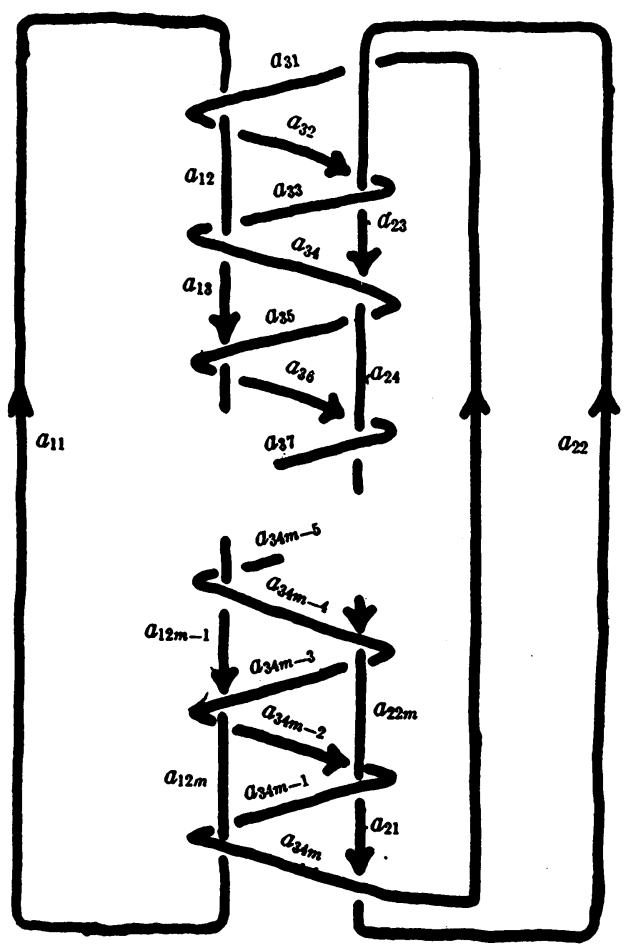

FIG. 1

CoRollary 1. $G /[G, G]$ is free abelian of rank $n$.

Corollary 2. $[G, G] /[[G, G], G]\left(=G_{2} / G_{3}\right)$ is isomorphic with an additive group generated by $x_{i j}, i \neq j, i, j=1,2, \cdots, n$, with relations

(a)

(b)

$$
\begin{aligned}
x_{i j}+x_{j i} & =0, i \neq j, & i, j & =1,2, \cdots, n, \\
\sum_{j=1, i \neq j}^{n} \mu_{i j} x_{i j} & =0, & i & =1,2, \cdots, n .
\end{aligned}
$$

Proof. Let $\Re^{*}$ be the normal subgroup generated by all $r_{i}^{*}$, 
$i=1,2, \cdots, n$, in $\mathfrak{F}$. We have $\Re^{*} \subset \mathfrak{F}_{2}$. It is straightforward that

$$
G_{2} / G_{3} \cong \mathfrak{S}_{2}^{*} / \mathbb{S}_{3}^{*} \cong \mathfrak{F}_{2} / \Re^{*} \cdot \mathfrak{F}_{3} \cong\left(\mathfrak{F}_{2} / \mathfrak{F}_{3}\right) /\left(\Re^{*} \cdot \mathfrak{F}_{3} / \mathfrak{F}_{3}\right) \text {. }
$$

$\mathfrak{F}_{2} / \mathfrak{F}_{3}$ is a free abelian group having as a basis the elements $\left[a_{i}, a_{j}\right]$ $\bmod \mathfrak{F}_{3}, i>j, i, j=1,2, \cdots, n . \Re^{*} \cdot \mathfrak{F}_{3} / \mathfrak{F}_{3}$ is a subgroup of $\mathfrak{F}_{2} / \mathfrak{F}_{3}$ and is generated by elements $\prod_{j=1, j \neq i}^{n}\left[a_{j}, a_{i}\right]^{\mu_{i j}} \bmod \mathfrak{F}_{3}$. Write the group $\mathfrak{F}_{2} / \mathfrak{F}_{3}$ additively, and replace $\left[a_{j}, a_{i}\right] \bmod \mathfrak{F}_{3}$ by $x_{i j}$. Thus the corollary follows immediately.

Corollary 3. If $L=L_{1} \cup L_{2}$, then $[G, G] /[[G, G], G]$ is cyclic of order $\left|\mu_{12}\right|$.

K. Reidemeister [7, p. 45] remarked that, for $L=L_{1} \cup L_{2},\left[a_{1}, a_{2}\right]$, taken as an element of $G / G_{3}$, is of order $\left|\mu_{12}\right|$. This result may be regarded as a corollary of Theorem 2 .

5. Examples. Let $L=L_{1} \cup L_{2} \cup L_{3}$ be a link as given in Fig. 1, and $G$ its group. The link $L$ has three components, each pair of which has vanishing linking number. We shall therefore overlook the factor group $G_{2} / G_{3}$, which does not yield interesting invariants. In order to compute $G_{3} / G_{4}$, let $F$ be the free group generated by $a_{i j}, i=1,2,3$; $j=1,2, \cdots, k_{i}, k_{1}=k_{2}=2 m, k_{3}=4 m$. Write, for $j=1,2, \cdots, m$,

$$
\begin{array}{ll}
b_{12 j-1}=a_{34 j-3}, & b_{12 j}=a_{34 j}^{-1} ; \\
b_{2 j-1}=a_{34 j-4}^{-1}, & b_{22 j}=a_{34 j-1} ; \\
b_{34 j-3}=a_{12 j}, & b_{34 j-2}=a_{2 j} ; \\
b_{34 j-1}=a_{12 j}^{-1}, & b_{34 j}=a_{22 j+2}^{-1} .
\end{array}
$$

Write $v_{i j}=\left[b_{i j}, a_{i j}\right]$ and $r_{i j}=v_{i j} a_{i j} a_{i j+1}^{-1}$. Then $G$ is presented by generators $a_{i j}$ and relations $r_{i j}, i=1,2,3 ; j=1,2, \cdots, k_{i}$. Define $a_{i}=a_{i 1}$; $u_{i 1}=e, u_{i j}=v_{i j-1} v_{i j-2} \cdots v_{i 1}, j \neq 1 ; h_{i j}=u_{i j} a_{i} a_{v}^{-1} ; r_{i}=v_{i}{ }^{\prime} k_{i} v_{i k_{i}-1}$ $\cdots v_{i 1}$. As given in the preceding section, $G$ may be presented by generators $a_{i j}$ and relations $h_{i j}$ and $r_{i}, i=1,2,3 ; j=1,2, \cdots, k_{i}$. Let $\mathbb{S} \cong\left\{a_{1}, a_{2}, a_{3} / \phi \psi\left(r_{1}\right), \phi \psi\left(r_{2}\right), \phi \psi\left(r_{3}\right)\right\}$. Then $G / G_{4} \cong(\$) / \$_{4}$, which implies $G_{3} / G_{4} \cong \mathrm{G}_{3} / \mathfrak{S H}_{4}$.

As before, $\mathfrak{F}$ denotes the free group generated by $a_{1}, a_{2}, a_{3}$. The following congruence identities may be verified straightforwardly: For any $u, u^{\prime}, v, w \in \mathfrak{F}$,

$$
\begin{aligned}
{\left[u u^{\prime}, v, w\right] } & \equiv[u, v, w]\left[u^{\prime}, v, w\right] \bmod \mathfrak{F}_{4}, \\
{\left[u^{-1}, v, w\right] } & \equiv[u, v, w]^{-1} \bmod \mathfrak{F}_{4}, \\
{\left[u^{-1}, v\right] } & \equiv[v, u, u][u, v]^{-1} \bmod \mathfrak{F}_{4}
\end{aligned}
$$


First we have

$$
\begin{aligned}
\phi \psi\left(a_{12 j-1}\right) & \equiv a_{1} \bmod \mathfrak{F}_{3}, \\
\phi \psi\left(a_{12 j}\right) & \equiv\left[a_{3}, a_{1}\right] a_{1} \bmod \mathfrak{F}_{3}, \\
\phi \psi\left(a_{22 j-1}\right) & \equiv a_{2} \bmod \mathfrak{F}_{3}, \\
\phi \psi\left(a_{22 j}\right) & \equiv\left[a_{3}, a_{2}\right]^{-1} a_{2} \bmod \mathfrak{F}_{3}, \\
\phi \psi\left(a_{34 j-3}\right) & \equiv a_{3} \bmod \mathfrak{F}_{3}, \\
\phi \psi\left(a_{34 j-2}\right) & \equiv\left[a_{1}, a_{3}\right] a_{3} \bmod \mathfrak{F}_{3}, \\
\phi \psi\left(a_{34 j-1}\right) & \equiv\left[a_{2}, a_{3}\right]\left[a_{1}, a_{3}\right] a_{3} \bmod \mathfrak{F}_{3}, \\
\phi \psi\left(a_{34 j}\right) & \equiv\left[a_{2}, a_{3}\right] a_{3} \bmod \mathfrak{F}_{3} .
\end{aligned}
$$

Using the above identities, we have

$$
\begin{aligned}
\phi \psi\left(v_{12 j-1}\right) & =\left[\phi\left(a_{34 j-3}\right), \phi\left(a_{12 j-1}\right)\right] \equiv\left[a_{3}, a_{1}\right] \bmod \mathfrak{F}_{4}, \\
\phi \psi\left(v_{12 j}\right) & =\left[\phi\left(a_{34 j}^{-1}\right), \phi\left(a_{12}\right)\right] \\
& \equiv\left[\left(\left[a_{2}, a_{3}\right] a_{3}\right)^{-1},\left[a_{3}, a_{1}\right] a_{1}\right] \bmod \mathfrak{F}_{4} \\
& \equiv\left[a_{2}, a_{3}, a_{1}\right]^{-1}\left[a_{3}, a_{1}\right]^{-1} \bmod \mathfrak{F}_{4}, \\
\phi \psi\left(v_{22 j-1}\right) & \equiv\left[a_{2}, a_{3}, a_{3}\right]\left[a_{2}, a_{3}, a_{2}\right]^{-1}\left[a_{3}, a_{2}\right]^{-1} \bmod \mathfrak{F}_{4}, \\
\phi \psi\left(v_{22}\right) & \equiv\left[a_{2}, a_{3}, a_{2}\right]\left[a_{1}, a_{3}, a_{2}\right]\left[a_{3}, a_{2}, a_{3}\right]\left[a_{3}, a_{2}\right] \bmod \mathfrak{F}_{4}, \\
\phi \psi\left(v_{34 j-8}\right) & \equiv\left[a_{3}, a_{1}, a_{3}\right]\left[a_{1}, a_{3}\right] \bmod \mathfrak{F}_{4}, \\
\phi \psi\left(v_{34-2}\right) & \equiv\left[a_{3}, a_{2}, a_{3}\right]^{-1}\left[a_{1}, a_{3}, a_{2}\right]^{-1}\left[a_{2}, a_{3}\right] \bmod \mathfrak{F}_{4}, \\
\phi \psi\left(v_{34-1}\right) & \equiv\left[a_{3}, a_{1}, a_{3}\right]^{-1}\left[a_{2}, a_{3}, a_{1}\right]\left[a_{1}, a_{3}\right]^{-1} \bmod \mathfrak{F}_{4}, \\
\phi \psi\left(v_{34 j}\right) & \equiv\left[a_{3}, a_{2}, a_{3}\right]\left[a_{2}, a_{3}\right]^{-1} \bmod \mathfrak{F}_{4},
\end{aligned}
$$

for $j=1,2, \cdots, m$. It follows that

$$
\begin{aligned}
\phi \psi\left(r_{1}\right) & \equiv\left(\phi \psi\left(v_{12} v_{12 j-1}\right)\right)^{m} \bmod \mathfrak{F}_{4} \\
& \equiv\left[a_{2}, a_{3}, a_{1}\right]^{-m} \bmod \mathfrak{F}_{4} \\
\phi \psi\left(r_{2}\right) & \equiv\left(\phi \psi\left(v_{2} v_{2} v_{2 j-1}\right)\right)^{m} \bmod \mathfrak{F}_{4} \\
& \equiv\left[a_{2}, a_{3}, a_{1}\right]^{m} \bmod \mathfrak{F}_{4}
\end{aligned}
$$

and

$$
\begin{aligned}
\phi \psi\left(r_{3}\right) & \equiv\left(\phi \psi\left(v_{2}{ }_{4 j} v_{3}{ }_{4 j-1} v_{3} 4_{4 j-2} v_{3}{ }_{4 j-3}\right)\right)^{m} \bmod \mathfrak{F}_{4} \\
& \equiv\left[a_{2}, a_{3}, a_{1}\right]^{m}\left[a_{1}, a_{3}, a_{2}\right]^{-m} \bmod \mathfrak{F}_{4} \\
& \equiv\left(\phi \psi\left(r_{1}\right)\right)^{-1}\left(\phi \psi\left(r_{2}\right)\right)^{-1} \bmod \mathfrak{F}_{4} .
\end{aligned}
$$

Now $\mathcal{S} \cong\left\{a_{1}, a_{2}, a_{3} / \phi \psi\left(r_{1}\right), \phi \psi\left(r_{2}\right), \phi \psi\left(r_{3}\right)\right\}$, and

$\mathbb{S} / \mathcal{S}_{4} \cong\left\{a_{1}, a_{2}, a_{3} / \phi \psi\left(r_{1}\right), \phi \psi\left(r_{2}\right), \phi \psi\left(r_{3}\right), \mathfrak{F}_{4}\right\}$. 
Thus, by Tietze operations,

$$
\text { S) } / \mathcal{S}_{4} \cong\left\{a_{1}, a_{2}, a_{3} /\left[a_{2}, a_{3}, a_{1}\right]^{m},\left[a_{1}, a_{3}, a_{2}\right]^{m}, \mathfrak{F}_{d}\right\} .
$$

Define $\mathcal{S}^{*} \cong\left\{a_{1}, a_{2}, a_{3} /\left[a_{2}, a_{3}, a_{1}\right]^{m},\left[a_{1}, a_{3}, a_{2}\right]^{m}\right\}$. Let $\Re^{*}$ be the normal

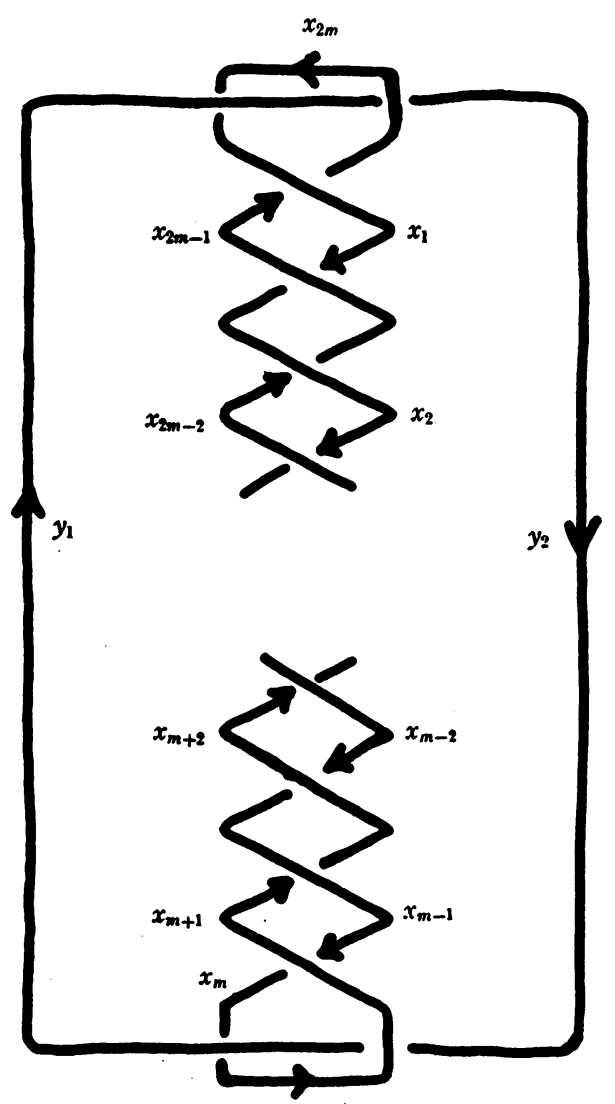

FIG. 2

subgroup generated by $\left[a_{2}, a_{3}, a_{1}\right]^{m}$ and $\left[a_{1}, a_{3}, a_{2}\right]^{m}$ in $\mathfrak{F}$. Then $\mathfrak{S}_{3} / \mathbb{S}_{4}$ $\equiv \mathfrak{F}_{3} / \mathfrak{R}^{*} \cdot \mathfrak{F}_{4} \equiv\left(\mathfrak{F}_{3} / \mathfrak{F}_{4}\right) /\left(\mathfrak{R}^{*} \cdot \mathfrak{F}_{4} / \mathfrak{F}_{4}\right)$. The group $\mathfrak{F}_{3} / \mathfrak{F}_{4}$ is free abelian of rank $8[2 ; 4 ; 9]$. We may choose as a basis for $\mathfrak{F}_{3} / \mathfrak{F}_{4}$ the elements $\left[a_{1}, a_{2}, a_{1}\right],\left[a_{1}, a_{2}, a_{2}\right],\left[a_{1}, a_{2}, a_{3}\right],\left[a_{1}, a_{3}, a_{1}\right],\left[a_{1}, a_{3}, a_{2}\right],\left[a_{1}, a_{3}, a_{3}\right]$, $\left[a_{2}, a_{3}, a_{2}\right],\left[a_{2}, a_{3}, a_{3}\right] \bmod \mathfrak{F}_{4}$. The group $\Re^{*} \cdot \mathfrak{F}_{4} / \mathfrak{F}_{4}$ is free abelian with $\left[a_{2}, a_{3}, a_{1}\right]^{m},\left[a_{1}, a_{3}, a_{2}\right]^{m} \bmod \mathfrak{F}_{4}$ as basis. Hence $G_{3} / G_{4}$ is isomorphic to a direct product $J_{m} \times J_{m} \times B_{6}$ where $J_{m}$ is a cyclic group of order $m$, and $B_{6}$ is a free abelian group of rank 6.

Consider another link $L=L_{1} \cup L_{2}$ (Fig. 2) which has a vanishing 
linking number. In order to avoid the extensive use of double indices, we write the group $G$ of $L$ in the presentation:

$$
G \cong\left\{x_{1}, x_{2}, \cdots, x_{2 m}, y_{1}, y_{2} / r_{1}, r_{2}, \cdots, r_{2 m}, s_{1}, s_{2}\right\}
$$

where

$$
\begin{aligned}
r_{i} & =\left[x_{2 m-i}, x_{i}\right] x_{i} x_{i+1}^{-1}, \\
r_{m} & =\left[y_{1}, x_{m}\right] x_{m} x_{m+1}^{-1}, \\
r_{2 m} & =\left[y_{1}^{-1}, x_{2 m}\right] x_{2 m} x_{1}^{-1}, \\
s_{1} & =\left[x_{2 m}^{-1}, y_{1}\right] y_{1} y_{2}^{-1}, \\
s_{2} & =\left[x_{m+1}, y_{2}\right] y_{2} y_{1}^{-1} .
\end{aligned}
$$

Let $F$ be the free group generated by $x_{1}, x_{2}, \cdots, x_{2 m}, y_{1}, y_{2}$, and denote $x=x_{1}, y=y_{1}, r=\left[y_{1}^{-1}, x_{2 m}\right]\left(\prod_{i=1}^{m-1}\left[x_{i}, x_{2 m-i}\right]\right)\left[y_{1}, x_{m}\right]$ - $\left(\prod_{i-m+1}^{2 m-1}\left[x_{i}, x_{2 m-i}\right]\right), s=\left[x_{m+1}, y_{2}\right]\left[x_{2 m}^{-1}, y_{1}\right]$. Define the substitutions $\psi$ and $\phi$ such that $\psi\left(x_{1}\right)=x, \psi\left(x_{i+1}\right)=r_{i} x_{i+1} x_{i}^{-1} \psi\left(x_{i}\right), i=1,2, \cdots$, $2 m-1, \psi\left(y_{1}\right)=y, \psi\left(y_{2}\right)=\left[x_{2 m}^{-1}, y\right] y, \phi\left(x_{i}\right)=x, \phi\left(y_{i}\right)=y$. Then, according to Theorem 1 ,

$$
G / G_{5} \cong\left\{x, y / \phi \psi^{2}(r), \phi \psi^{2}(s), \mathfrak{F}_{5}\right\}
$$

where $\mathfrak{F}$ is the free group generated by $x$ and $y$. We are going to show that $\phi \psi^{2}(r) \equiv[x, y, x, y]^{m-1} \bmod \mathfrak{F}_{5}$ and $\phi \psi^{2}(s) \equiv[x, y, x, y]^{-m+1} \bmod \mathfrak{F}_{5}$, which will imply that

$$
G / G_{5} \cong\left\{x, y /[x, y, x, y]^{m-1}, \mathfrak{F}_{5}\right\} .
$$

From $[u, a v]=[u, a] a[u, v] a^{-1}$ it follows that, if $u \in \mathfrak{F}_{s}, v \in \mathfrak{F}_{t}, a \in \mathfrak{F}_{q}$, then $[u, a v] \equiv[u, a][u, v] \bmod \mathfrak{F}_{s+t+q}$. The above congruence identity will be used frequently in this example.

It is immediate that $x=\phi \psi\left(x_{1}\right)=\phi \psi\left(x_{2}\right)=\cdots=\phi \psi\left(x_{m}\right)$ and $[y, x] x=\phi \psi\left(x_{m+1}\right)=\cdots=\phi \psi\left(x_{2 m}\right)$. It follows that, for $i=1,2, \cdots$, $m-1$,

$$
\phi \psi\left(\left[x_{2 m-i}, x_{i}\right]\right) \equiv[[y, x] x, x] \equiv[y, x, x] \bmod \mathfrak{F}_{4},
$$

and, for $i=m+1, \cdots, 2 m-1$,

$$
\phi \psi\left(\left[x_{2 m-i}, x_{i}\right]\right) \equiv[y, x, x]^{-1} \bmod \mathfrak{F}_{4} .
$$

By the definition of $\psi$,

$$
\psi\left(x_{m}\right)=\left(\prod_{i=1}^{m-1}\left[x_{m+i}, x_{m-i}\right]\right) x
$$

and 


$$
\psi\left(x_{2 m}\right)=\left(\prod_{i=1}^{m-1}\left[x_{i}, x_{2 m-i}\right]\right)\left[y, x_{m}\right] \psi\left(x_{m}\right) .
$$

Thus we have

$$
\phi \psi^{2}\left(x_{m}\right) \equiv[y, x, x]^{m-1} x \bmod \mathfrak{F}_{4}
$$

and

$\phi \psi^{2}\left(x_{2 m}\right) \equiv[y, x, x]^{-m+1}\left[y, \phi \psi\left(x_{m}\right)\right][y, x, x]^{m-1} x \equiv[y, x] x \bmod \mathfrak{F}_{4}$.

Since $\psi\left(\left[x_{2 m-i}, x_{i}\right]\right) \in F_{3}$ and $\psi\left(\left[y_{1}, x_{m}\right]\right) \in F_{2}$,

$$
\begin{aligned}
\psi(r) & \equiv \psi\left(\left[y_{1}^{-1}, x_{2 m}\right]\right)\left(\prod_{i=1}^{m-1}\left[x_{i}, x_{2 m-i}\right]\right) \psi\left(\left[y_{1}, x_{m}\right]\right) \psi\left(\prod_{i=1}^{m-1}\left[x_{i}, x_{2 m-i}\right]\right)^{-1} \\
& \equiv \psi\left(\left[y^{-1}, x_{2 m}\right]\right) \psi\left(\left[y, x_{m}\right]\right) \bmod F_{5} .
\end{aligned}
$$

Consequently

$$
\begin{aligned}
\phi \psi^{2}(r) & \equiv\left[y^{-1}, \phi \psi^{2}\left(x_{2 m}\right)\right]\left[y, \phi \psi^{2}\left(x_{m}\right)\right] \\
& \equiv\left[y^{-1},[y, x] x\right]\left[y,[y, x, x]^{m-1} x\right] \\
& \equiv[x, y][x, y, x, y]^{m-1}[y, x] \equiv[x, y, x, y]^{m-1} \bmod \mathfrak{F}_{5}
\end{aligned}
$$

On the other hand, $\psi\left(y_{2}\right)=\left[x_{2 m}^{-1}, y\right] y, \phi \psi^{2}\left(y_{2}\right)=\left[\phi \psi\left(x_{2 m}^{-1}\right), y\right] y \equiv\left[z^{-1}, y\right] y$ $\bmod \mathfrak{F}_{4}$, where $z=[y, x] x$. Moreover $\psi\left(x_{m+1}\right)=\left[y, x_{m}\right] \psi\left(x_{m}\right)$, and $\phi \psi^{2}\left(x_{m+1}\right)=\left[y, \phi \psi\left(x_{m}\right)\right] \phi \psi^{2}\left(x_{m}\right) \equiv[y, x][y, x, x]^{m-1} x \equiv[y, x, x]^{m-1} z$ $\bmod \mathfrak{F}_{4}$. Thus

$$
\begin{aligned}
\phi \psi^{2}(s) & \equiv\left[[y, x, x]^{m-1} z,\left[z^{-1}, y\right] y\right]\left[z^{-1}, y\right] \\
& \equiv\left[[y, x, x]^{m-1}, y\right]\left[z,\left[z^{-1}, y\right] y\right]\left[z^{-1}, y\right] \\
& \equiv[y, x, x, y]^{m-1}\left[y, z^{-1}\right]\left[z^{-1}, y\right] \\
& \equiv[x, y, x, y]^{-m+1} \bmod \mathfrak{F}_{5} .
\end{aligned}
$$

Therefore $G / G_{5} \cong\left\{x, y /[x, y, x, y]^{m-1}, \mathfrak{F}_{5}\right\}$. The factor group $\mathfrak{F}_{4} / \mathfrak{F}_{5}$ is a free abelian group of rank 3 . As its basis, we may choose $[x, y, x, x]$, $[x, y, x, y],[x, y, y, y] \bmod \mathfrak{F}_{5}[4]$. The factor group $G_{4} / G_{5}$ is hence abelian and isomorphic with a direct product $J_{m-1} \times B_{2}$ where $J_{m-1}$ is the cyclic group of order $m-1$, and $B_{2}$ is a free abelian group of rank 2 , and the integer $m-1$ is a numerical invariant of the link.

\section{BibliogRAPHY}

1. R. Baer, The higher commutator subgroups of a group, Bull. Amer. Math. Soc. vol. 50 (1944) pp. 143-160.

2. K. T. Chen, Integration in free groups, Ann. of Math. vol. 54 (1951) pp. 147-162.

3. R. H. Fox, Free differentiation, forthcoming. 
4. M. Hall, $A$ basis for free Lie ring and higher commutators in free groups, Proceedings of the American Mathematical Society vol. 1 (1950) pp. 575-581.

5. W. Magnus, Beziehungen zwischen Gruppen und Idealen in einem speziellen Ring, Math. Ann. vol. 111 (1935) pp. 259-280.

6. - Über freie Faktorgruppen und freie Untergruppen gegebener Gruppen, Monatshefte für Mathematik und Physik vol. 47 (1939) pp. 307-313.

7. K. Reidemeister, Knotentheorie, Berlin, Springer, 1932.

8. - Einfiuhrung in die kombinatorische Topologie, Braunschweig, Vieweg, 1932, pp. 46-49.

9. W. Witt, Treue Darstellung der Lieschen Ringe, J. Reine Angew. Math. vol. 177 (1937) pp. 152-160.

Princeton University 